\title{
Entre azulejos y rejas, entre el arte y la arquitectura: el ornamento en la ciudad y en la frontera
}

\author{
FERNANDA GUIMARAES GOULART \\ Profesora de la Escuela de Bellas Artes. Departamento de Diseño \\ Universidad Federal Minas Gerais, Brasil \\ nandagg@uol.com.br
}

\begin{abstract}
Resumen
Este texto presenta una parte de mi investigación de doctorado "Urbano Ornamento: un inventario de rejas ornamentales en Belo Horizonte (y otras bellezas)" con respecto al estudio de viviendas que combinan rejas y azulejos ornamentales, que he llamado "Casas-Patchwork". Lo interesante es que son azulejos que se encuentran a menudo en las cocinas o cuartos de baño, lo que refuerza un cierto estatus de improvisación. Como metodología, intento acercarme del objeto a través de nociones que interesan, de manera concomitante, a los campos críticos del Arte y de la Arquitectura. En contacto constante a lo largo de su historia, estos campos son vistos aquí como máquinas reflexivas, capaces de hacer pensar sobre la temporalidad compleja de las ciudades, los modos sobrepuestos de vivir y habitar, la imaginación como una alternativa al olvido. Por consiguiente, la fachada es vista aquí como un lugar de frontera entre el espacio privado y doméstico, capaz de hacer emerger sentidos de movilidad y porosidad, tanto en relación a la presencia del ornamento en la ciudad actual, cuanto acerca de las maneras de acceder al objeto teóricamente. Para ambos, texto y ciudad, hay que identificar o reclamar una mirada poética que, si por un lado puede apuntar a un criterio científico aparentemente arbitrario, por otro lado puede ampliar las posibilidades de comprenderlos y experimentarlos.
\end{abstract}

Palabras clave: Ornamento; grade; azulejo; fachada; frontera. 


\title{
Between tiles and grilles, between art and architec- ture: ornament in the city and the border
}

\begin{abstract}
This paper presents a part of my doctoral research "Urban ornament: an inventory of ornamental railings in Belo Horizonte (and other beauties)" according with the study of houses that combine ornamental railings and tiles, which I called "Patchwork Houses ". The curious thing is that those tiles are often found in kitchens or bathrooms, reinforcing a kind of improvisation status. As a methodology, I attempt to approach the object through notions that matter, concomitantly, to the critical fields of Art and Architecture. In constant contact throughout their history, these fields are seen here as reflexive machines, increasing reflections about the complex temporality of cities, superposed modes of living and inhabiting, the imagination as an alternative to oblivion. By conseguinte, the facade is seen here as a border between the private and domestic space, abble to do emerge senses of mobility and porosity, both in relation to the presence of ornament in the city today, as about ways of accessing the object theoretically. For both, text and city, is important to identify or claim a poetic view, that if one side can point to a seemingly arbitrary scientific approach, on the other hand can expand the possibilities of understanding and experience them.
\end{abstract}

Key words: Ornaments; grille; tile; facade; border.

Son pequeñas casas de retazo, aquellas. Juntas podrían formar una colcha colorida, hecha a mano y enredada en las redes de la ciudad. Colcha abstracta, patchwork que sólo existiría en una u otra imaginación. O entre sí, en una muda conversación que sus superficies ornamentadas -encajadas, bordadas, cosidas, improvisadas- por ventura promuevan. Durante mucho tiempo, mi curiosidad quería adentrarse dentro de cada una que había visto, averiguar si el dentro correspondería a la atención dada al afuera, saber quiénes eran las personas que optaron por hacerlo así, tan femeninas a nuestros ojos. Sin embargo limité a recibirlas sólo como superficie, dejar que hagan parte de un museo imaginario, el que intento construir ahora. Sobre todo porque sus 
habitantes, o muchos de ellos, probablemente ya no serían los mismos, y yo no quería despertar la indiferencia a lo que a mí me parecía un puro pertenecer. ¿Tiene sentido intentar salvar una belleza casi invisible de la desaparición? ¿Cómo equiparar sus valores de improvisación y copia, en los hogares de ayer y de hoy (el tiempo requerido por el hacer, el pensar, y el desear...)? Pues hay otro tipo de saturación en la ciudad, que tiene que ver con una especie de ceguera en la que no se puede ver nada en medio al exceso y a la estetización y funcionalización de la publicidad. ¿Podrá ser el ornamento todavía un catalizador para otras miradas y percepciones en la ciudad?

Por patchwork comúnmente se entiende una unión de varias piezas de tejido cosidos entre sí, formando colchas, almohadas y otros elementos de paño, generalmente multicoloridos, alegres, femeninos. Estas casas tienen en común azulejos decorados en las fachadas, muchos de ellos con patrones de flores y colores claros. Lo curioso es que son patrones de azulejos que se encuentran a menudo en las cocinas o cuartos de baño, lo que refuerza un cierto estatus de improvisación, propio de una "arquitectura espontánea". Las casas tienen vinculadas a sus azulejos rejas ornamentales, instaladas en puertas y ventanas que abren en tales superficies coloridas intervalos llenos de delicadas líneas de hiefro. mayoría de estas casas cuenta con una u otra característica moderna, que anuncia y retrocede, tal vez atestando la singularidad de un cierto modernismo brasileño, más en sintonía con la tradición, modernismo que se empeña en no dejar lo ornamental. El diseño de muchas de ellas es geometrizado, asociado a otras características: parapetos rectangulares, horizontales o en escalera, ocultando el techo; caídas de techo diagonales tipo "mariposa", reflejadas o asimétricos; columnas de barras de hierro decorativas, diagonales y verticales; terrazas y garajes que equilibran llenos y vacíos; presencia de "cobogós" en las fachadas creando áreas de composición; vergas en relieve que enmarcan las ventanas por encima y por debajo, entre otros. Algunos apuntan a una proto-modernidad que trata de hacer, aunque no conscientemente, una transición entre Deco y el moderno, otras son típicos ejemplos modernos. Una especie de "modernidad periférica", y a veces kitsch, como dirían Dinah Guimaraens y Lauro Cavalvanti (2006). 
Sospecho que hay un interior que se revela a través de la fachada, condición de frontera. Transparente y móvil, como las que hacen las estatuas se confundieren con la propia ciudad, en "Saber de Pedra", de Luis Alberto Brandão. $\mathrm{O}$, de lo contrario, fronteras que son líneas gruesas, objetos mismos, como he entendido atestaren las voces minerales: La unidad de las cosas no está en las cosas mismas, sino en los cortes que en ellas tenemos la intención de hacer (Brandão, 1999: 68) También hay las que no sólo dejan de afastar como acercan, negando la separación entre los espacios urbanos y domésticos, imantando una interioridad en el contexto de la calle, quizá permitiendo afuera lo que no se puede permitir en su interior. Así es que la frontera se revela, de modo inquietante pero implacable, como puente, establecimiento de vecindad (Brandão, 2005: 78).

Fachada como ropa de la casa, casi piel.

Qué imagen de concentración el hecho de que esta casa se 'apreta' contra sus habitantes, convirtiéndose en una célula de un cuerpo, con sus paredes cercanas. El refugio se contrajo. Y, muchísimo protector, se hizo aparentemente más fuerte (Bachelard, 1974: 49)

Pienso en las inversiones bachelardianas, de la casa, de la ciudad y del mundo: casa que es mi piel y mi mundo, ciudad que es mi casa y casa que habita el mundo. Reconozco aquí el hombre y el espacio doméstico mezclados, confundidos, y también la falta de distinción entre materia y subjetividad, en la medida que se hace difícil de captar, como se nos alerta Ludmila Brandao -al parecer como consecuencia de Gaston Bachelard- lo que de la casa no es aquél que le habita.

Entre los hombres y las casas algo pasa más o menos así. Las casas son ciudades (y las ciudades, casas), destilan olores propios, sino que también son sus hombres, al igual que sus animales, objetos, sus historias y sus delirios.

Entre los hombres y las casas, ciertamente sin abdicar de una distinción mínima que no ofenda la razón, se procesa encuentro especial. De la naturaleza íntima. (Brandão, 2002: 134).

Antônio Carlos Leite Brandão (2011), por su parte, especula sobre la posibilidad de expresión de la arquitectura que no pase por la subjetividad, pero que sea capaz, por sí misma - en una perspectiva albertiana, y por lo 
tanto humanística, es importante recordar - singularizar el espacio, "dar forma al vacío", retirarse y ceder el paso, espacializar "el ser y el ser-otro posible". Sin embargo, aunque no subjetiva, es la condición de la arquitectura ser espacio existencial -al modo de Norberg-Schulz- porque, como quiere el autor:

Vivimos siempre entre un interior y un exterior, entre un espacio y otros, entre lo familiar y lo extraño, bajo techos y paredes, pero también delante del exterior que las puertas y ventanas nos permiten atravesar y llegar, con sus peligros y aventuras que dan sentido al doméstico y a la familia, entre el fuera y el dentro, entre el yo y el otro, entre las cuatro paredes que nos dan protección y la visibilidad pública que nos pone en juego (...) Se habita, y ahí está la arquitectura, siempre un espacio de travesía, que los lugares neutros, sin durée y homogéneos, como los aeropuertos y las ciudades homogéneas, como Euroville, no nos permiten experimentar (Brandão, 2011: 4).

Así parece explicar doña Carmélia, residente del barrio Sagrada Familia, en Belo Horizonte/Brasil, para quien su casa abarca toda la calle. Yo vivo en la calle, ella no termina en mis límites. Mi hábitat es extenso! Yo vivo pajeando la calle.. $\mathrm{Al}$ igual que la señora Carmélia se relaciona con la vecindad, su casa lo hace. Si ella habla a través de la ventana, con la vecina que pasa por la calle, con los nietos que viven en la planta de abajo, con las aves, con su perro, su casa debe hacer lo mismo, de acuerdo con ella.

La imagen de frontera también aparece en Paul Ricoeur (1998), para quien los lugares siempre son un pasaje, por dónde se pasa y a donde se llega. Es en este sentido que el habitar, así como el construir, tienen movilidad, al contrario de lo que la estabilidad de la "piedra" podría hacer, a través de su "permanecer", "parar", "fijar". Es en este sentido también que la casa y la ciudad, la arquitectura y el urbanismo, participan, al menos filosóficamente, de la misma dinámica.

Habitar implica ritmos de paradas y movimientos, fijación y desplazamientos. El lugar no es sólo la cavidad donde fijarse, tal como se lo definía Aristóteles (la superficie interior del sobre), sino también el intervalo a recorrer. La ciudad es el primer sobre desta dialéctica de abrigo y desplazamiento (Ricoeur, 1998: 4). 
En su ensayo "Arquitectura y narratividad", Ricoeur compara la arquitectura a la actividad narrativa, y expresa su intertextualidad, cuando el habitar no es sólo pre-arquitectura - en el momento en que empezamos a preparar el espacio, lo que el autor llama de un "habitar-construir primordial" - o construcción -la "configuración" del habitar, arquitectura en sí, que incluye el proyecto, con todo el ideario de su época-, pero la respuesta, llena de capas. Es en este punto que podemos entender el assemblage de tiempos que la ciudad nos da a leer, también cuando el ornamento se reviste de la temporalidad efímera y constantemente actualizable del presente. Vuelvo a citar a Ricoeur:

$Y$ de la misma manera que la recepción del texto literario inaugura el experimento de una lectura plural, de una acogida paciente hecha a la intertextualidad, también el habitar receptivo y activo implica una relectura cuidadosa del ambiente urbano, un nuevo y constante reaprendizaje de la yuxtaposición de los estilos, y por lo tanto también de las historias de vida que marcan edificios y monumentos. Hacer con que estos rasgos no sean sólo residuos, sino testimonios reactualizados de un pasado que ya no existe, pero que ya fue, hacer con que el haber-sido del pasado sea salvo a pesar de su noser-más; es lo que puede la "piedra" que dura (Ricoeur, 1998: 9).

¿Qué idioma hablan estas fachadas? ¿Cómo son capaces de exteriorizar domesticidades? ¿Qué historias nos cuentan, sobre las ciudades, las casas y el habitar de los hombres? ¿Es posible promover una escucha de este "saber de frontera", no sólo a través de las huellas e impresiones de cada casa, pero de la atmósfera y del paisaje configurado por su conjunto? Ludmila Brandao nos alerta sobre el desafío de reconocer a cada día la conjunción de elementos dispares -sonidos, olores, experiencias, eventos, olvido- que componen el doméstico. Así que tal vez sea necesario imaginar que se trata de una frontera en movimiento y hacer vibrar y retumbar su porosidad.

Fronteras que se les deberán identificarse también en las tensiones entre el arte y la arquitectura. Sucintamente y luego podemos mencionar algunos de esas tensiones: la vieja idea de integración entre las artes, bajo la égida de la madre arquitectura; su carácter plástico y escultórico, al ejemplo del arte; las experiencias con el espacio-obra (que no deja de ser arquitectónico) y el cuerpo, del hombre y de la arquitectura; las derivas y recorridos por la 
ciudad, que ni siempre requieren la mediación del arte para ser experimentados estéticamente; la cara crítica de la arquitectura que se materializa en proyectos, discursos, instalaciones, y en ellos se le sacia. Tales relaciones no sólo se ocupan (lo que no es pequeño ni de fácil comprensión) de representar -como lo hacen los dos campos independientes-, sino de presentar poética y políticamente un problema, vibrátil y táctilmente una experiencia. Pero Stéphane Huchet nos advierte:

Hoy en día, la resistencia contra las nuevas formas de integración mutua entre la arquitectura y el arte es un problema porque, como dialécticas intraplásticas, esas formas de integración son sólo la versión contemporánea de la gran demanda histórica y cultural de la arquitectura: fundar, conformar y configurar el mundo (Huchet, 2005: 178).

En este sentido, es conveniente recordar que los artistas no raramente intentan conocer como los sujetos viven y construyen la urbe -sobre todo modos no dominados por la Ingeniería Civil, el Urbanismo y de otras 'estructuras de poder', por así decirlo- que apuntan a la singularidad de las experiencias en las ciudades, en dirección contraria al espectáculo y a la especulación inmobiliaria. Llamo la atención sobre la relación entre el arte y la vida cotidiana, tan cara al discurso artístico contemporáneo y muy presente en esta reflexión, sobre el gesto mientras trivial y estético de adornar la fachada. Sospecho que la manifestación de la subjetividad expresada en el "habitar" implica cambios recíprocos entre las dimensiones ordinarias y estética de la vida, más allá de lo es puramente decorativo, sin necesidad de alcanzar lo que sea ontológicamente artístico $^{1}$. Es como si la dimensión de la experiencia, temporal y afectiva, estuviese en un lugar más estético -si no atraemos esta cualidad a lo que es de orden superficial- que artístico o plástico -donde estarían el diseño arquitectónico y la obra de arte-. Ese "habitar" el trabajo parece ser, en ese sentido, circunstancial, mientras lo de la casa se muestra cotidiano, así que nos queda entender cómo la Estética como disciplina -en su sesgo relacional, sin duda-

\footnotetext{
${ }^{1}$ La elección del término "dimensión estética" en lugar de "dimensión artística" se debe al hecho de que consideramos el primer más amplio que el segundo, que sólo puede referirse a las obras de arte, ya que queremos hablar de la extensión del sentido decurrente de ciertas experiencias de los sujetos en el mundo.
} 
puede ser comprendida ahí. Fronteras que deben ser identificadas también con respecto a un campo de plástico, como quiere Stéphane Huchet (2005).

El campo plástico, por lo tanto, encarna un valor que estructura tanto las bellas artes como la arquitectura misma. Si la historia de la relación entre la arquitectura y el arte no ha escapado a la reproducción y perpetuación de puntos de vista convencionales sobre ellos, el gran logro de un enfoque crítico es la posibilidad de desarmar irreductibilidades recíprocas, conectadas a la rigidez de las categorías, $y$ de abrir sus polos a cambios e intercambios experimentales y transdisciplinarios (Huchet, 2005: 172).

Consideremos estos "intercambios experimentales y transdisciplinarios", de que habla Huchet, través de la fuerza gráfica de las rejas y azulejos. Es que la presencia del diseño en el espacio urbano también imprime esa mirada a través de un sentido formal (que es, sin embargo, crítico) más allá de la cáscara estilística, llenando la ciudad de ayer y de hoy con colores y líneas, dando gracia discreta a los caminos, con tono distinto de los muros de vidrio de hoy y los alambres de espino rizados. Aquí se vislumbra la voluntad de abstracción de todo ornamento - condicionado por su geometría inmanente y caro a su constitución histórica - bien como otra grieta, que muestra su condición de escrita, una "escritura natural de la humanidad" a René Smeets (1982). En el caso del dibujo, un "mundo sin subtítulos", al que se refiere Edith Derdyk: Lineas que se proyectan en el espacio universal, provocando tesituras siempre emergentes y en tránsito (Derdyk, 2007: 24). Diseñar para no olvidar, Edith aprendió un hombre analfabeto, que recitaba versos de memoria.

En este estudio, la línea es sobre todo metáfora de la frontera, pero punteada, porosa: entre el arte y la arquitectura, y entre la casa y la calle. También es una línea de costura, para conformar un mosaico urbano y multitemporal. Es línea voluptuosa, que genera movimiento y responde por el impulso ornamental, erótico y de proliferación. Y es aún línea de proyecto, para conformar una arquitectura ilustrada, señalando la importancia histórica del dibujo para este campo de conocimiento, desde los tratados: una escrita compartida, cuando el arquitecto se va "más allá del lenguaje" (escrito) (Evers, 2003). Por último, la metáfora de la línea-limite, que es también delimite, y sigue trazando el campo de la arquitectura, algo parecido a lo Ludmila Brandao escribe, o dibuja: 
El fenómeno arquitectónico (...) Yo prefiero pensarlo como un espacio desmesurado -tal vez como aquél del plan que consiste en la espesura de una hoja de papel- uno entre dos fueras, donde todo sucede. (...) Quizás porque sea cómo la arquitectura y sus cortes a-significativos: tan tenues los limites, como la espesura de la hoja de papel, se compone según un plan que acoge sólo líneas, intensidades (Brandão, 2002: 34).

Ludmila Brandao adentra por su "casa subjetiva" de modo curioso, con ese adjetivo y frase. Curioso. -escribe- Para escribir acerca de esas casas fue necesario olvidar la arquitectura. Fue necesario, sobre todo al principio (y no sin culpa y vergüenza), descalificar la arquitectura como oficio y sus procedimientos más habituales, teóricos y prácticos (Brandão, 2002: 4). Fue necesario hacer de la arquitectura una "indisciplina", que es construida por el vano, dice. Vale todavía traer lo que la autora señala como "insuficiencia" del campo de la arquitectura, en cuanto a la relación entre la teoría y la práctica, entre diseño y experiencia, podríamos decir. Me refiero a la máquina reflexiva que el arte parece tener éxito en poner a trabajar, lo que permite el conocimiento no sólo de la vida ordinaria, sino que de la ciudad, del pasado, de la memoria. Parece que esto suele ser una "responsabilidad" más del artista que del propio arquitecto, otro temas en que pensar. Ludmila también nos recuerda que la mayoría de los estudios de arquitectura están respaldados por perspectivas históricas, sociológicas o antropológicas. Artísticas también, se puede añadir.

Tal vez no tenemos que ir a Venecia para aprender con Ruskin a tener atención al alma de las calles, las casas, los pequeños detalles y a mirar algunos trabajos ornamentales como focos privilegiados de resistencia al desencantamiento del mundo (Paim, 2000: 36) Bastaría comprender su intención de representar, y qué. Si el monumento es la excepción, la casa es nota normal de la vida cotidiana del ciudadano, es como una lápida epigráfica de su ascendencia y de su historia (apud Pinheiro \& D'Agostino, 2004: 124). Como se expresa Mariano Filho sobre una casa colonial en Río de Janeiro: Todo en él es verdad. Todo tiene su razón de ser, su lógica, su significado (Idem). Y Lucio Costa, rendido a una cierta verdad marginal: Al ver aquellas casas, aquellas iglesias, de sorpresa en sorpresa, nosotros como que nos encontramos, quedamos contentos, felices y recordamos cosas olvidadas, cosas que nunca supimos, pero que estaban dentro de nosotros, no lo sé (Pinheiro \& D'Agostino, 2004: 125). 
Parece que estas "permanencias subterráneas", en las palabras de Huchet (2004a), que las vanguardias no se las pueden negar, o al menos olvidarlas, son una especie de fantasma, un asombro perenne. Si hablamos de la arquitectura espontánea, así que tal vez no vamos a encontrar "piedra sobre piedra". ¿Pero lo que queda de la batalla contra el ornamento? ¿Es posible eliminar la capa de la ideología que hace opaco el ornamento? ¿Qué sentido sobrevive a este conflicto hoy tan historiado? Tal vez sea una batalla tan anacrónica a nuestros ojos como la insistencia de estas casitas en colorear el paisaje de la metrópoli.

Hay un artículo de Andreas Huyssen (1996), cuyo título es "La cultura de masas como mujer", que subraya las inscripciones de género con respecto a la relación dialéctica entre la vanguardia y la industria cultural. Su pretexto es Madame Bovary, personaje de Flaubert, aficionada por la literatura inferior, con sus dosis desequilibradas de subjetividad, emoción y pasividad. El escritor, por su parte, es el hombre, el creador de la literatura auténtica, objetiva, irónica, controlada en sus formas estéticas. Pero Flaubert personalmente confesó que él y Bovary eran la misma persona. Está pautada por asociaciones semejantes la relación entre la vanguardia (el macho) y la cultura de masas (la mujer). Alentado por argumentos como los de una alta cultura misógina que coloca a las mujeres en una posición inferior (máximo como fuente de inspiración), Huyssen dirán que el femenino, el inconsciente, la sexualidad, la debilidad, amenazan: las masas son la esfinge que devora a través de la mercantilización y el tipo equivocado de éxito. No sólo él, sino también la diversión y el placer.

¿Se hizo con placer? ¿Hacemos eco de la voz de Ruskin aquí? Aunque los argumentos son muy diferentes (si bien ambos terminan frente a la crítica de la explotación del trabajo), dejemos el binomio racionalidad/intuición alimentar conceptos y lemas modernos -autenticidad, autoridad, autoría, auto-referencia, conciencia, ideal- tal vez para ver cómo pueden permanecer intactos los sistemas significantes de la vida cotidiana. He aquí un conjunto que es selva oscura y que escapa al control, refracta las comparaciones, la raíz del sublime (Pinheiro \& D'Agostino, 2004: 122), frente a la autoridad indiscutible de la modernidad en el rediseño del escenario de la vida (Huchet, 2004a: 74).

Invita Huyssen (1996: 44) a concluir: El hombre, después de todo, puede negar su propia subjetividad, en beneficio de una meta estética más elevada, 
ya que su existencia se garantiza en el nivel de la experiencia cotidiana. Por lo tanto, Christa Wolf concluye, con algunas dudas, pero sin perder la fuerza: $\mathrm{La}$ estética como la filosofía y la ciencia, se crean no tanto para que podamos acercarnos a la realidad, pero para distraernos, para protegernos de ella.

\section{Bibliografía}

ANGOtTI-SAlgueIro, H. (1987): O Ecletismo em Minas Gerais: Belo Horizonte 1894-1930. En FABRIS, A. (Org.). Ecletismo na Arquitetura Brasileira. Nobel/Edusp, São Paulo, págs. 104-145.

BRANDÃO, Carlos Alberto (2011): “A cidade não é uma obra de arte". Conferência de conclusão do curso Gestão do Patrimônio Cultural e Ambiental da UFMG. Tiradentes, 26/02/2011, Hotel Mãe D’Água, UFMG.

BRANDÃO, Ludmila (2002): A casa subjetiva: matérias, afetos e espaços domésticos. Ed. Perspectiva, São Paulo.

BRANDÃO, Luis Alberto (1999). Saber de Pedra. O livro das estátuas. Autêntica, Belo Horizonte.

GUIMARES, Dinah y CAVALCANTI, Lauro (2006): Arquitetura kitsch suburbana e rural. Paz e Terra, Rio de Janeiro.

HUCHET, Stéphane (2005): Horizonte tectônico e campo "plástico" - de Gottfried Semper ao Grupo Archigram - pequena genealogia fragmentária. En MAlARD, Maria Lúcia (org.) (2005): Cinco textos sobre arquitetura. Editora UFMG, Belo Horizonte.

HUYSSEN, Andreas (1996): Memórias do modernismo. Editora UFRJ, Rio de Janeiro.

PAIM, Gilberto (2000): A beleza sob suspeita: o ornamento em Ruskin, Lloyd Wright, Loos, Le Corbusier e outros. Jorge Zahar Editor, Rio de Janeiro.

PINHeIRO, Maria Lucia Bressan y D’Agostino, Mário Henrique (2004): A noção de pitoresco no debate cultural das primeiras décadas do século XX no Brasil. Desígnio, revista de arquitetura e urbanismo. Faculdade de Arquitetura e Urbanismo da Universidade de São Paulo, FAU/USP, no ${ }^{1}$, marzo de 2004, págs. 119-127.

RICOEUR, Paul (1988): Arquitetura e Narratividade. Urbanism, no 303, noviembre/diciembre de 1988, págs. 44-51. 